\title{
E. Fazit
}

Im Zweiten Teil dieser Arbeit hat sich die Tragfähigkeit der im Ersten Teil unter F aufgestellten Thesen erwiesen. Anhand konkreter Beispiele strafrechtlicher Begriffsrelativität konnte gezeigt werden, dass bedeutungsdivergierende Verwendungen beziehungsweise Auslegungen buchstäblich identischer Ausdrücke nicht generell einen Verstoß gegen das Gesetzlichkeitsprinzip des Art. 103 Abs. 2 GG bedeuten, dies im Einzelfall aber sehr wohl der Fall sein kann. Das gilt für die Verwendungen auf Rechtssetzungsebene und die Auslegungen auf der Ebene der Rechtsanwendung gleichermaßen.

Des Weiteren hat sich bestätigt, dass es bei der strafrechtlichen Begriffsrelativität im Hinblick auf bedeutungsunterschiedliche Auslegungen identischer Zeichenketten regelmäßig nicht um Fälle von Homonymie, sondern um solche der Extension und Restriktion geht, weshalb angesichts des Analogieverbotes die Prüfung dahingehend zu erfolgen hat, wann eine extensive oder restriktive Interpretation in eine Analogie beziehungsweise eine Reduktion umschlägt, mithin die Grenze zulässiger Auslegung überschritten wird, wobei ein Verfassungsverstoß nur dann vorliegt, wenn es sich um ein täterbelastendes Ergebnis handelt. Die im Vorangegangen behandelten konkreten Beispiele haben gezeigt, dass es im Rahmen einer solchen Prüfung nicht zuletzt darauf ankommt, welche der verschiedenen Ansichten vom richtigen Verständnis der Wortlautgrenze dieser Prüfung zugrunde zu legen ist, im Einzelfall aber auch alle Ansichten zu dem gleichen Ergebnis führen können. Dabei vermitteln holistische Ansätze, die die Berücksichtigung sämtlicher konkreter Auslegungskontexte - insbesondere des teleologischen - im Rahmen der Wortlautgrenzziehung ermöglichen, der strafrechtlichen Begriffsrelativität die größten Spielräume. 